\title{
Multiple Criteria Decision Making, Multiattribute Utility Theory: Recent Accomplishments and What Lies Ahead
}

\author{
JYRKI WALLENIUS, JAMES S. DYER, PETER C. FISHBURN, RALPH E. STEUER, \\ STANLEY ZIONTS and KALYANMOY DEB
}

\begin{abstract}
Helsinki School of Economics, POB 1210, 00101 Helsinki, Finland, College of Business Administration, University of Texas, Austin, Texas 78712

AT\&T Laboratories, Florham Park, New Jersey 07932

Terry College of Business, University of Georgia, Athens, Georgia 30602

School of Management, State University of New York, Buffalo, New York 14260

Indian Institute of Technology Kanpur, UP 208016, India
\end{abstract}

\begin{abstract}
This article is an update of an article five of us published in 1992. The areas of Multiple Criteria Decision Making (MCDM) and Multiattribute Utility Theory (MAUT) continue to be active areas of management science research and application. This paper extends the history of these areas and discusses topics we believe to be important for the future of these fields.
\end{abstract}

Key words: decision making; multiattribute; multiple criteria

Acknowledgments: The authors wish to thank Craig W. Kirkwood, Arizona State University, Murat Köksalan, METU, and Roman Slowinski, Poznan University of Technology, as well as two anonymous reviewers for valuable comments.

\section{Introduction}

About fifteen years ago, five of us (Dyer, Fishburn, Steuer, Wallenius and Zionts, 1992) published a paper exploring the next decade for Multiple Criteria Decision Making (MCDM) and Multiattribute Utility Theory (MAUT). The purpose of the present paper is to review what has been done since that paper was written, and to outline interesting future research questions. Much has happened since that paper was published (see below); this has led to increased researcher mobility and to a more open exchange among researchers around the world concerning theory, software, and applications. Some of the 
more important events that have occurred and trends that have developed since 1992 that relate to the MCDM/MAUT fields include the following:

1. Use of the Internet has exploded and computing power has continued to grow, as evidenced by what is commonly referred to as Moore's Law. ${ }^{1}$ This has affected our fields and offers intriguing possibilities for the future. We now have web-based software for assisting users (even consumers) in applying MCDM/MAUT approaches.

2. Substantial growth in applications of MCDM/MAUT has occurred. See for example the website for Expert Choice: http://www.expertchoice.com ${ }^{2}$, the recent reviews of applications of decision analysis by Keefer et al. (2004) and Hämäläinen (2004), the survey of applications of multiobjective evolutionary algorithms by Coello and Lamont (2004), and the results of our scientometric analysis of published papers.

3. The importance of MCDM/MAUT has been recognized in professional management journals. See the article about Thomas Saaty in FORTUNE (1999) and the article on "even swaps" as a rational way of making tradeoffs in the Harvard Business Review (Hammond et al. 1998).

4. The importance of behavioral aspects of decision making has grown, and this was recognized by the award of the 2002 Nobel Prize in Economics to Daniel Kahneman. It is widely believed that his late colleague Amos Tversky would have shared this honor.

5. Data envelopment analysis (DEA) has grown in importance and its relationship with multiple objective linear programming (MOLP) has been explored.

6. Evolutionary multiobjective optimization (EMO) has emerged as a new field with strong ties to MCDM/MAUT.

7. Heuristics in MCDM/MAUT have become more important.

${ }^{1}$ According to Pew Internet \& American Life Project, a non-profit research center studying the social effects of the Internet on Americans, in a survey conducted in February-March 2007, 69\% of adult Americans used the Internet on an average day, $41 \%$ used a search engine to find information, $56 \%$ to send or read email. Obviously the statistics are quite different for third world countries.

${ }^{2}$ Weblinks may change over time. We will try to provide names of individuals to contact, should the links no longer be active. 
8. MCDM/MAUT has begun to penetrate many new areas of research and applications.

MCDM/MAUT has several neighboring disciplines, such as decision analysis, mathematical programming, DEA, and negotiation analysis. MCDM/MAUT concepts and methods are being developed and used in these neighboring disciplines, providing healthy cross-fertilization, but making it difficult to draw sharp disciplinary boundaries. In this paper we assess how MCDM/MAUT has interfaced with these neighboring disciplines. Another interesting trend is that MCDM/MAUT concepts and techniques are increasingly being applied in diverse engineering fields and other application areas.

Our objectives are to: a) review what has happened since the earlier paper was written; and b) identify exciting directions for future research. Although this paper is not an exhaustive survey, we include many additional references published since our earlier paper was published. Our paper is oriented towards methodological dimensions rather than application domains, because generic methodological advances can be employed across a broad spectrum of applications.

\section{Our Decision-Making Framework}

We assume a decision maker ${ }^{3}$ who chooses one (or a subset) of a set of alternatives evaluated on the basis of two or more criteria or attributes. The feasible set of solutions may be either small and finite (as in choice problems) or large and perhaps infinite (as in design problems). Uncertainty may be involved. Conceptually, we may assume that a decision maker acts to maximize a utility or value function that depends on the criteria or attributes. In cases of uncertainty, the problem is typically to maximize the expected value of a utility function. We believe that an important part - possibly the most important part of MCDM/MAUT is the support of decision making in a broader sense. MCDM/MAUT methods are intended to help a decision maker think about the problem as part of the decision-making process.

There are two categories of MCDM/MAUT problems: multiple criteria discrete alternative problems and multiple criteria optimization problems. Examples of discrete alternative problems include

\footnotetext{
${ }^{3}$ The decision maker may be an individual or a group that cooperates to act according to the same rational decision making process as one that would be followed by an individual. In some cases we will also include a discussion of how MCDM/MAUT methods may assist with more complex multi-person decision situations such as those encountered in negotiations.
} 
choosing the location for a new airport, selection of a computer network, choice of a drug rehabilitation program, and identifying which nuclear power plant to decommission. They are "discrete" alternative problems because sets of alternatives typically consist of modestly-sized collections of choices, although an emerging class of discrete problems called multiple criteria sorting problems may have hundreds of alternatives (see Doumpos and Zopounidis, 2002).

Examples of optimization problems include river basin planning, energy planning, engineering component design, portfolio selection, and $R \& D$ project selection. Feasible sets of alternatives for such problems usually consist of a very large or infinitely many alternatives, defined by systems of equations and inequalities that identify the feasible region for the decision variables. Because the vectors of alternatives may have many components and the number of equations and inequalities may be large, the feasible regions may be complex. Consequently, multiple criteria optimization problems are likely to require relatively more computational resources than discrete problems.

In addition to differences in the feasible sets of alternatives, there are other differences between multiple criteria discrete alternative and multiple criteria optimization problems. One is that discrete alternative problems are more likely to be modeled with uncertain values for the attributes or criteria, than multiple criteria optimization problems. Another difference is in the way utility or value functions are taken into account. Many approaches to multiple criteria discrete problems attempt to represent aspects of a decision maker's utility or value function mathematically and then apply these results to estimate the alternatives' (expected) utilities. In multiple criteria optimization, there is usually no attempt to capture the decision maker's utility or value function mathematically. Rather the philosophy is to iteratively elicit and use implicit information about the decision maker's preferences in order to help steer the decision maker to his most preferred solution.

By implicit information we mean answers to questions such as (a) which of two solutions is more preferred, (b) which of several tradeoff vectors is most preferred, and (c) which criterion values can be relaxed in order to allow improvements in other criteria. If the elicited information enables an interactive 
procedure to converge to a "final solution" in a reasonable number of iterations, then the final solution is one that is either optimal (most preferred), or good enough to terminate the decision process.

Because of different problem types, different families of approaches have evolved for solving discrete alternative problems and multiple criteria optimization problems. Topmost among the approaches for solving discrete alternative problems is MAUT as described by Keeney and Raiffa (1976). MAUT and the Analytic Hierarchy Process (AHP), as described in Saaty (1999), would basically constitute what some European scholars call the American school, whereas methods based on a partial ordering of alternatives such as ELECTRE (in fact a family of methods) and PROMETHEE are examples of the French school. The emphasis on different international schools is unfortunate because it makes it difficult to classify approaches developed by international teams (see Olson, 1996). Approaches for solving multiple criteria optimization problems fall into the following categories: interactive methods, goal programming, vector-maximum algorithms, and evolutionary procedures, although these categories are not necessarily mutually exclusive. For technical discussions of these and other specific MCDM/MAUT methods, see for example, Edwards et al.(2007), Figueira et al. (2005), Ignizio and Romero (2003), and Caballero et al. (2007).

\section{Bibliometric Analysis of MCDM/MAUT}

We have conducted a basic bibliometric study of MCDM/MAUT using the ISI database. The ISI database, which covers over 8650 journals including a broad range of disciplines, is updated on a weekly basis. We report basic statistics regarding how our fields have developed based on variations of the following key words: multiple criteria decision, multiattribute utility, multiple objective programming/optimization, goal programming, Analytic Hierarchy Process, evolutionary/genetic multiobjective, and vector optimization.

Figures 1 and 2 show the number of publications and the number of citations of papers in our fields, respectively, over the 1970-2006 period and until the end of June 2007. Growth in the number of publications and in the number of citations been rapid since 1992 when our earlier paper was published, and has increased even more dramatically since 2000. 
Roughly $10 \%$ of the publications have appeared in EJOR, with about $1 \%$ each in Management Science and in Operations Research. The remainder are widely scattered over many different journals. TABLES 1 and 2 provide information about publications by country of residence of the first author, and sub-topical areas within the MCDM/MAUT fields. Although authors from the United States have been most prolific (30.3\% of the total), the other $69.7 \%$ have come from all over the world underscoring the international nature of MCDM/MAUT research. Among the sub-topical areas within MCDM/MAUT, the environment is listed fifth reflecting its application potential. Also, engineering and computer science are important reflecting the broad, interdisciplinary nature of our fields.

FIGURE 3 provides information by methodological area. The growth in AHP-related publications is enormous, as is the recent growth in EMO publications. By contrast, goal programming and mathematical programming have maintained more stable patterns of growth. The research related to the "French School" may have been underestimated because of our difficulty in finding appropriate keywords.

\section{FIGURE 1}

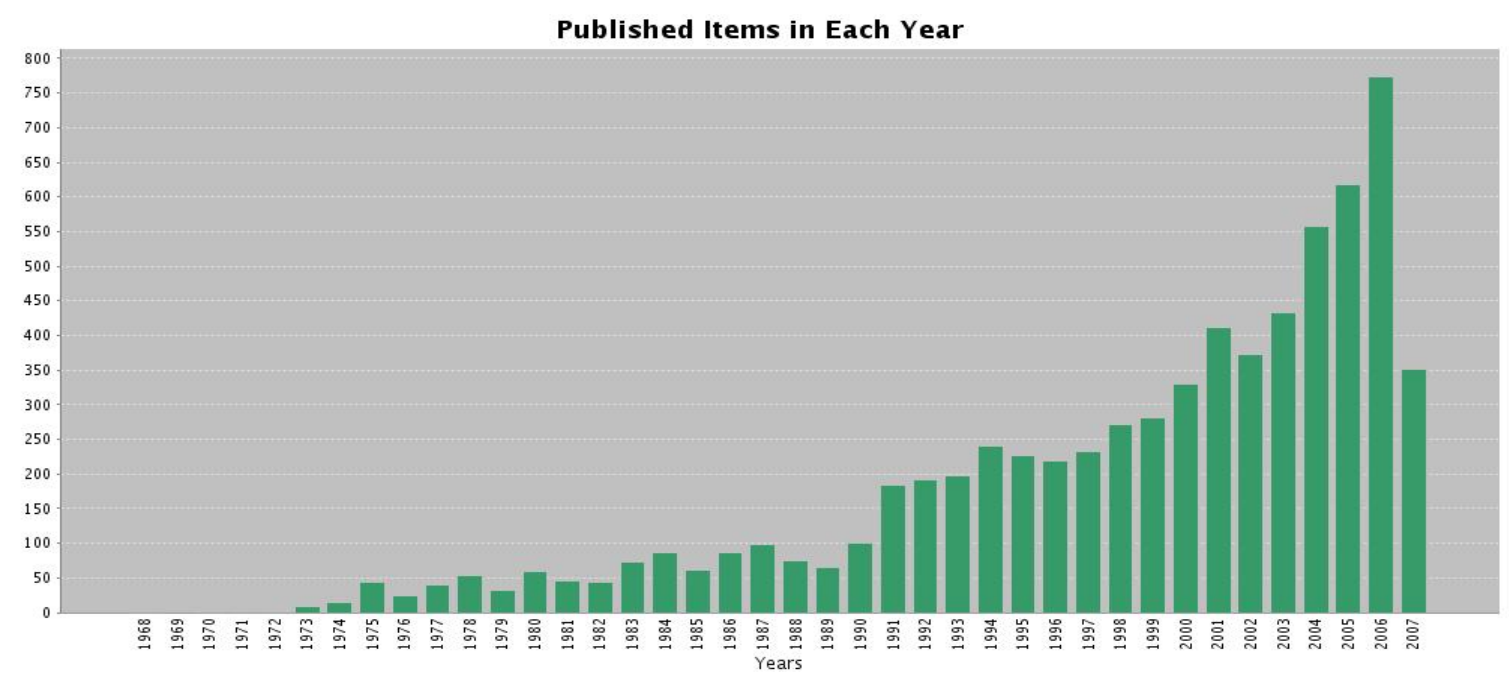

Note: year 2007 includes published items until the end of June, 2007 


\section{FIGURE 2}

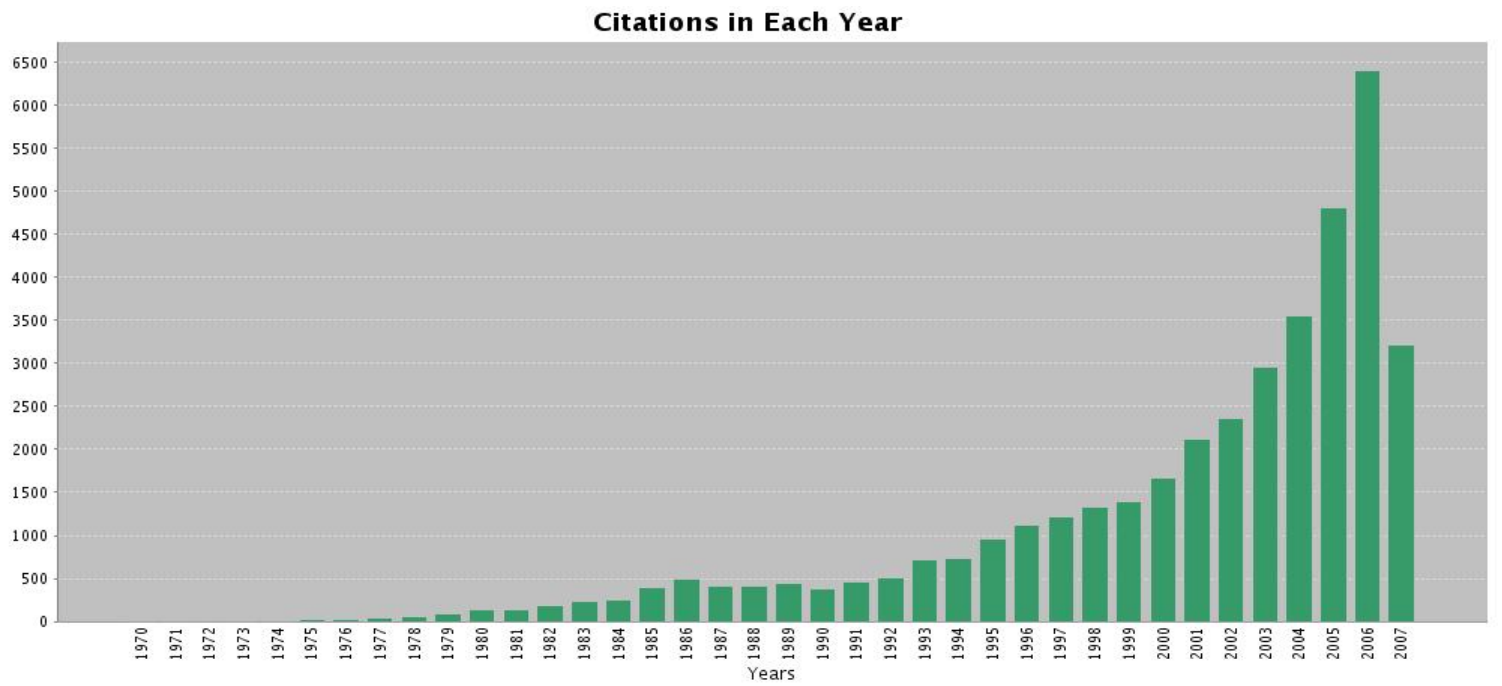

Note: year 2007 includes citations until the end of June, 2007

\section{TABLE 1: Publications by Country}

\begin{tabular}{|l|r|r|}
\hline Country & Numbers & $\%$ \\
\hline USA & 2097 & 30.3 \\
\hline China & 471 & 6.8 \\
\hline England & 441 & 6.4 \\
\hline Canada & 351 & 5.1 \\
\hline Taiwan & 329 & 4.8 \\
\hline Spain & 306 & 4.4 \\
\hline India & 302 & 4.4 \\
\hline Germany & 264 & 3.8 \\
\hline Japan & 241 & 3.5 \\
\hline Italy & 235 & 3.4 \\
\hline Australia & 202 & 2.9 \\
\hline France & 195 & 2.8 \\
\hline South Korea & 189 & 2.7 \\
\hline Finland & 184 & 2.7 \\
\hline Netherlands & 176 & 2.5 \\
\hline Others & 927 & 13.4 \\
\hline Total & 6910 & 100 \\
\hline
\end{tabular}

\section{TABLE 2: Sub-topical Areas}

\begin{tabular}{|l|r|r|}
\hline $\begin{array}{l}\text { Operations research and } \\
\text { management science }\end{array}$ & 2415 & $34.9 \%$ \\
\hline $\begin{array}{l}\text { Computer science and artificial } \\
\text { intelligence }\end{array}$ & 1829 & $26.5 \%$ \\
\hline Management and business & 1587 & $23.0 \%$ \\
\hline $\begin{array}{l}\text { Applied mathematics, } \\
\text { interdisciplinary }\end{array}$ & 1066 & $15.4 \%$ \\
\hline Environmental & 689 & $10.0 \%$ \\
\hline Industrial engineering & 641 & $9.3 \%$ \\
\hline Manufacturing engineering & 405 & $5.9 \%$ \\
\hline Economics & 308 & $4.5 \%$ \\
\hline Civil Engineering & 289 & $4.2 \%$ \\
\hline $\begin{array}{l}\text { Computer science and information } \\
\text { systems }\end{array}$ & 270 & $3.9 \%$ \\
\hline Energy and water resources & 267 & $3.9 \%$ \\
\hline
\end{tabular}


FIGURE 3 Publication History: Area of Research

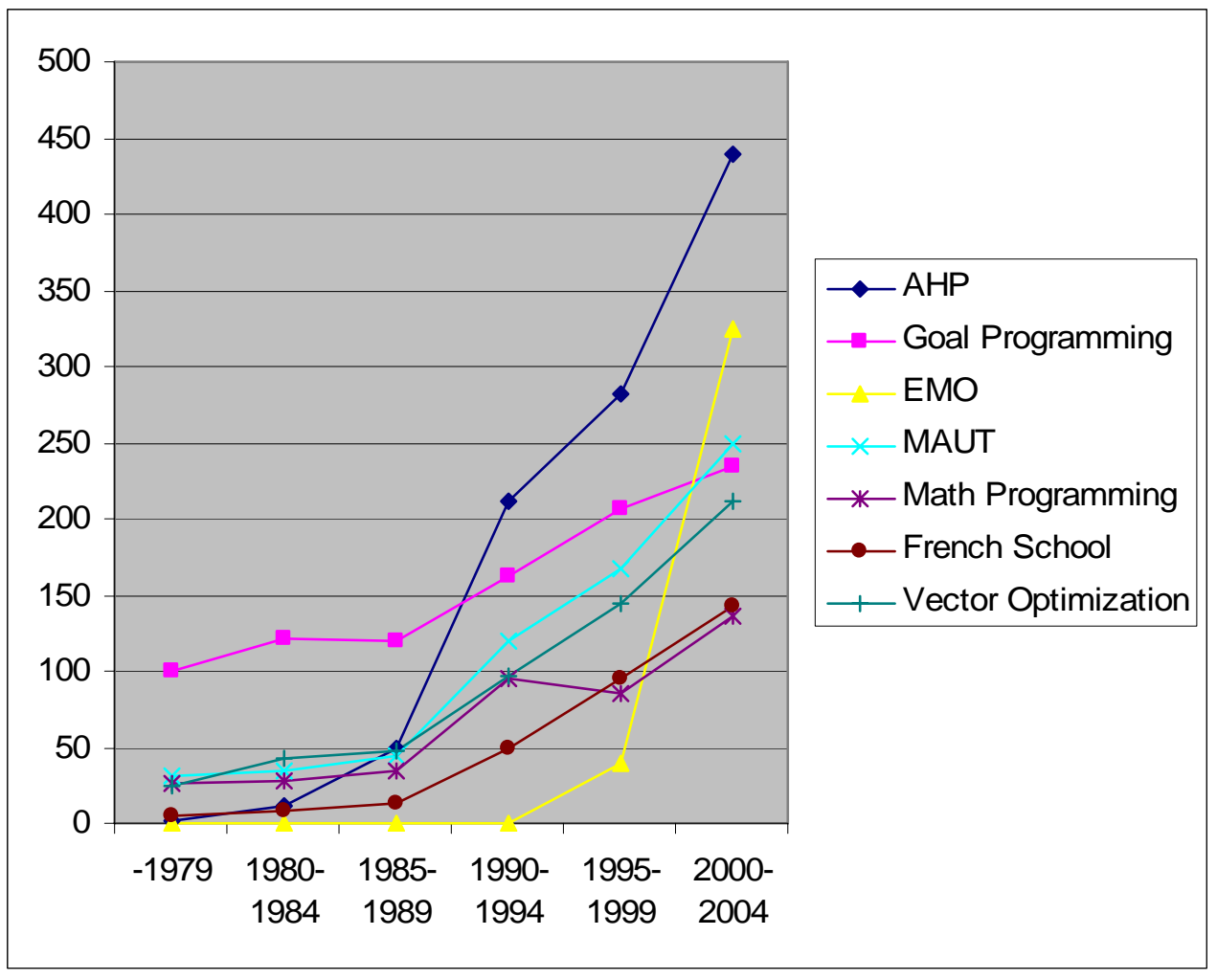

\section{Contributions since Our 1992 Paper}

In this section we focus on the evolution of the MCDM $\backslash$ MAUT fields since our earlier paper. We begin with a review of the areas that we identified as opportunities for future research, and then discuss other changes in these fields that we failed to emphasize or anticipate.

\subsection{Areas of future research identified in 1992}

Our 1992 paper identified four major MCDM/MAUT areas where we predicted significant future research contributions, namely: decision support applications, incorporation of behavioral research, emphasis on robust decisions, and the role of heuristics.

These four areas have experienced significant research productivity, although some more than others. However, work related to heuristics has outperformed our expectations because its growth came from a source that was not expected; it has grown dramatically because of EMO.

In the early 1990's we could foresee the continuing spread of personal computers, and we could predict the popularity of spreadsheet modeling and spreadsheet solvers. We believe that we also 
anticipated the upcoming advances in computing power, but not necessarily all the consequences. But we did not know about the popularity and the importance of the web-protocol. Tim Berners-Lee originally developed "hypertext" and the first Web server for CERN at the end of 1980s, and by 1991 the World Wide Web (WWW) as we know it today was born. Its significance became apparent a few years later. It is now influencing many aspects of human life, and is presenting great challenges and opportunities for our profession. We comment on its significance in several places.

Another development that we did not foresee is the penetration of MCDM/MAUT modeling into engineering, electronic commerce, DEA, and negotiation science. This is exciting, but a concern is that many scholars who apply MCDM/MAUT tools and concepts in their own disciplines may lack knowledge about existing MCDM/MAUT methods. As a result, they may rediscover work that has already been done or they may not be aware of useful ideas.

\section{Decision Support Applications of MCDM/MAUT}

The business world has become more competitive and less predictable, accentuating the importance of effective decision making and the use of decision support tools. The decision support systems that are widely used are user friendly and often employ spreadsheets, such as Excel, at least for data entry. In fact, most OR/MS textbooks, which typically include a chapter on MCDM/MAUT, are now built around spreadsheets. In early work (certainly in the 1970’s and early 1980's) it was necessary to do significant custom programming to carry out applications. However, by 1997, one could usually accomplish essentially the same goals with a small amount of Visual Basic for Applications (VBA) programming in Excel. (See Kirkwood, 1997 and Ragsdale, 2004, on how to carry out such analyses in Excel using VBA and Solver.) The following web pages provide examples of MCDM/MAUT software applications: http://www.decisionarium.tkk.fi/, http://faculty.fuqua.duke.edu/daweb/dasw.htm/, http://www.logicaldecisions. com/, http://www.krysalis.co.uk/index.html/, http://www.logicaldecisions.com/, http://www.krysalis.co.uk/ index.html/, and http://www.strata-decision.com/.

Similarly, the invention of the Internet has created a need for additional decision support (in a distributed or even a mobile environment). Many scholars have implemented their favorite 
MCDM/MAUT procedures on the Internet. For example, the eclectic AIM method (Lotfi et al. 1992) now has a web version, WebAIM: http:// mgt2.buffalo.edu/webaim/. See Wang and Zionts (2005). One of the earliest examples of a web-based MCDM/MAUT decision support tool is WWW-NIMBUS (Miettinen and Mäkelä, 1995).

Decision support is also available for consumers online (for example, Active Decisions discussed below) and for decision conferencing. Advances in MAUT methods to support decision conferencing have been facilitated by the increases in readily available computing power. See Keefer et al. (2004), the work by Phillips et al. (http://www.catalyze.co.uk/), and the work by Hämäläinen et al. (http://www.decisionarium.tkk.fi/) for discussions of applications.

\section{Behavioral Considerations}

Behavioral issues have not received a great deal of attention by MCDM/MAUT researchers in recent years, despite the recognition of this topic and calls for further research by Herbert Simon in the 1950's. Korhonen and Wallenius (1996) provide of survey of salient behavioral issues and outline a 12-point research agenda emphasizing the design of interactive decision tools.

Recent work related to behavioral issues includes possible biases in the elicitation of weights (Keeney, 2002, Delquie, 1993, 1997, and Pöyhönen and Hämäläinen, 2001), and issues related to the design of value trees and the selection of attributes (e.g., Hämäläinen and Alaja, 2003). Related work may offer additional opportunities for research. These include studies that explore whether knowledge of alternatives and other contextual issues will bias assessments of the importance of attributes (see, for example, Carlson and Pearo, 2004, and Carlson and Bond, 2006).

Other research related to behavioral issues include an evaluation of alternative strategies for group decision making and issues related to organizational decision making. See Hämäläinen (2004) for a discussion of some MCDM/MAUT group decision making approaches and for some suggestions for research agendas related to them. Matheson and Matheson (2007) discuss organizational issues associated with the application of decision support models. Furthermore, problem structuring continues to 
be of significant interest. See for example Kirkwood (1997) and Ragsdale (2004) and the recent special issue of the Journal of the Operational Research Society (Vol. 57, Issue 7, 2006) on problem structuring. Scheubrein and Zionts (2006) have demonstrated the importance of a front-end (or problem formulation) system for solving MCDM problems.

\section{Robustness Considerations}

Robustness can be defined in many different ways. One can talk about robust decisions, robust algorithms, or robust optimization in a deterministic or probabilistic setting. Generally speaking, robustness refers to the ability of a solution to cope with uncertainties including those that may not be anticipated. Many papers have been published in recent years that deal with robustness considerations.

Hogarth and Karelaia (2005) provide a recent example of this work for MAUT. For examples of MCDM robustness research, see Roy (1998), Kouvelis and Sayin (2006), Deb and Gupta (2005), and Wang and Zionts (2006), along with the interesting work on rough sets, based on Pawlak's original idea (Pawlak, 1982; see also Slowinski, 1992, and the discusssion in section 4.2 below). Furthermore, many interval-valued methods provide robust solution approaches, including PAIRS for value tree analysis (Salo and Hämäläinen, 1992) and Robust Portfolio Modeling (Liesiö et al., 2007).

\section{Role of Heuristics}

Heuristics have become more important in recent years, particularly in the MCDM literature. Many realworld problems are so complex that one cannot reasonably expect to find an exact optimal solution. One broad field involved with such problems is multiple objective combinatorial optimization, which attempts to address multiple criteria knapsack, traveling salesman, and scheduling problems. Exact solution methods are augmented by simulated annealing, tabu search, and local search techniques. The interested reader is referred to the growing literature on this subject as illustrated by Ulungu and Teghem (1994), Ehrgott and Gandibleux (2000), Jaszkiewicz (2001), and Sun (2003). The rapidly-growing area of evolutionary procedures is related to the role of heuristics, but is discussed later.

\subsection{Computer Related Topics}


Increases in computing power have been at the heart of many of the advances in MCDM/MAUT. Along with algorithmic advances, larger and more complex problems are now solvable in reasonable time. On the MCDM side, this has had a major impact on procedures used for solving multiple-objective linear, multiple-objective nonlinear, and multiple-objective combinatorial optimization problems.

\section{Impact of Increased Computational Power}

As an MCDM-oriented benchmark, we use the case of computing all nondominated extreme points of a multiple objective linear program (MOLP) in order to obtain a discretized representation of its nondominated solution set. In the early 1990's, problems with only a few thousand nondominated extreme points were the largest that could be solved. Since the nondominated set grows exponentially with the number of objectives, this placed upper limits on the problems that could be solved at approximately 40 constraints by 80 variables with 3 objectives, 30 constraints by 60 variables with 4 objectives, and so forth. Now with faster computers and improved algorithms, problems with approximately one million nondominated extreme points can be solved in about the same amount of time, thus extending the size of MOLPs amenable to vector-maximum algorithms, such as Steuer's ADBASE (2006), to problems with hundreds of constraints and variables.

Algorithms for computing all nondominated facets of a feasible region are still of interest, but because of the computation involved they have yet to find a place in practice. Help in this regard may come from the normal vector identification ideas set forth in Yang and Li (2002). Also, as suggested in Wiecek and Zhang (1997), procedures are being developed for partitioning an MOLP so that the task of computing all nondominated extreme point solutions can be subdivided among parallel processors. This will enable the computation of MOLPs with millions of nondominated extreme points.

When a nondominated set is described by a large number of points, the task of finding a best or acceptable solution still remains. The nondominated set is a portion of the surface of the feasible region in criterion space. The dimensionality of the feasible region in criterion space is never greater than the number of objectives, but there may be several hundred thousand points representing a nondominated 
surface that would be like grains of sand on a beach. The challenge then is how to work one's way through the grains of sand to find the best one.

Interactive procedures may be used to enable a decision maker to move to better and better solutions, while learning much about the problem through interactions with points from the nondominated set. Descriptions of prominent interactive procedures are given in Gardiner and Steuer (1994) and Miettinen (1999). Although designed primarily for continuous case problems, many of them can be modified for navigating through large numbers of discrete solution points. In fact, Korhonen and Karaivanova (1999) have presented an algorithm which is being extended to handle more than a million discrete points.

Although MAUT does not in general have the computational demands of MCDM, the main benefit of advances in computing power has been to facilitate more responsive user-friendly interfaces with greater functionalities. This has led to a substantial increase in applications of MAUT methods, particularly those involved in web-based decision support and decision conferencing.

\section{Evolutionary Multicriterion Optimization (EMO)}

Despite prior advances, multiple criteria optimization techniques were unable to solve many highly nonlinear multiple criteria problems that were beginning to crop up in earnest, mostly in engineering, in the early 90s. While the Russian PSI (parameter space investigation) method of Statnikov and Matusov (2002) was earlier designed for some of these problems, an entirely new approach - one drawn from genetic algorithms -- called evolutionary algorithms (EAs) has been developed. Schaffer (1984) suggested the first multi-objective evolutionary algorithm called VEGA. Roughly ten years later three working evolutionary algorithms were suggested by three different groups of researchers: Fonseca and Fleming's (1993) MOGA, Srinivas and Deb's (1994) NSGA, and Horn et al.'s (1995) NPGA. This research helped spur the development of evolutionary multi-objective computation.

Starting with an initial population, an EA updates the population by using processes designed to mimic natural survival-of-the-fittest principles and genetic variation operators to improve the average population from generation to generation in a stochastic manner. The goal is to converge on a diverse 
final population of points that represents the nondominated set. EAs are suitable for use on parallel processors thereby enhancing their potential. The intriguing ideas of EAs were developed by people, mostly from engineering and computing, who had not formerly been connected with the MCDM/MAUT community. Representative references on EMO are provided by Corne et al. (2000), Branke, et al.(2001), Zitzler et al.(2001), Deb (2001 and 2003), Fonseca et al. (2003), and Coello and Lamont (2004).

\section{Knowledge Discovery, Preference Modeling}

Machine learning and knowledge discovery techniques have entered the field of preference modeling, mainly as a result of the concept of dominance-based rough sets (Greco et al., 1999, 2005). Slowinski et al. (2002) have provided axioms that differentiate among the main families of preference models: utility functions, outranking relations and decision rules. Preference learning from a sample of past decisions leads to a preference model, expressed as a set of "if - then" decision rules that can be used for descriptive as well as prescriptive purposes. An important feature of the Dominance-Based Rough Set Approach (DRSA) is the possibility of handling inconsistencies in past decisions. The DRSA concept allows classification of the decision rules into "certain" and "doubtful" rules, the latter resulting from

inconsistencies. DRSA, based on the rational principle of dominance, exploits ordinal properties of evaluations solely and seeks to be transparent to users. In addition to theoretical research, the rough sets research community has vigorously sought to apply its ideas for solving practical problems. Preference learning underlines and strengthens the links between artificial intelligence and MCDM.

\subsection{New Application Areas of MCDM/MAUT Methods}

MCDM methods and ideas have been integrated into new areas such as Data Envelopment Analysis (DEA), negotiation science, electronic commerce, spatial modeling, and, on a broad level, engineering. There is every reason to believe that this trend will continue. We now review some of these developments.

DEA

Charnes and Cooper conducted the pioneering research both in goal programming and Data Envelopment Analysis (Charnes and Cooper, 1961; Charnes et al., 1978). Yet, MCDM and DEA developed separately. 
Belton (1992) and Doyle and Green (1993) described the relationships between the two. Subsequently, Joro et al. (1998) developed a detailed understanding of the structural (mathematical) relationship between DEA and MOLP, and noted the close similarities that exist. One of the basic differences is the radial projection used in DEA and the more general nonradial projection used in MOLP. In other words, DEA mechanically (radially) extends the ray from the origin via the point representing the Decision Making Unit being evaluated to the efficient envelope when calculating efficiency scores. MOLP techniques are more generic and can be used to identify benchmarking units on the efficient frontier.

Of course, DEA and MOLP usually have different purposes. DEA is used for performance measurement, whereas MOLP is used for aiding choice. The observation about the structural similarity between DEA and MOLP has sparked synergistic advances in both models. For example, MOLP can be used to find nonradially projected targets on the efficient frontier (Korhonen and Syrjänen, 2004). Originally DEA did not consider the preferences of the decision maker. This created problems, because a unit could be efficient by being best in terms of one output measure, which could be the least important from the DM's point of view. As a way to incorporate preferences into DEA, researchers have suggested the use of weight restrictions. MOLP models can be used to generate novel ways of incorporating DM's preferences into DEA (Halme et al., 1999). On the other hand, DEA provides new application areas for MOLP researchers.

\section{Negotiation Science}

The literature on negotiation and group decision making is broad and diverse. The field is multidisciplinary, involving different approaches by social psychologists, economists and management scientists. A few common threads have become accepted in all these areas. One of them is the concept of a contract curve. It was introduced by economists, refined in game theory, and then re-worked by social psychologists. Later, management scientists developed models attempting to locate solutions on the contract curve. Other common threads include the assumption of limited negotiator rationality and the misrepresentation of negotiators’ preferences. 
Raiffa (1982) is a pioneer in this field. For a more recent discussion of negotiation theory and its relationship to decision analysis and game theory, see Sebenius (2007). Following Raiffa's footsteps, several MCDM/MAUT researchers have contributed to this problem area. Teich et al. (1994) classify modeling aids as follows: (1) whether or not an attempt is made to explicitly construct the participants' value functions; (2) whether participants are requested to make concessions from their preferred positions or whether we seek to identify jointly preferred (“win-win”) alternatives from a Single Negotiating Text (Fisher, 1978). This classification leads to four different modeling aids:

(1) value function and concession based models

(2) value function based and Pareto improvement seeking models

(3) interactive models based on concession making

(4) interactive models seeking Pareto improvements.

Each category offers interesting research opportunities. Ideas and tools have been picked eclectically from MCDM/MAUT. See for example Ehtamo et al. (1999) and Ehtamo and Hämäläinen’s review paper (2001). The problem area is rich, and includes novel applications of web-based negotiations. Wang and Zionts (2007) have developed an approach for quantifying certain aspects of negotiations and have shown that it is generally better to negotiate on multiple alternatives at the same time. Yet, documented practical applications are few.

\section{e-Commerce: Multi-Attribute Auctions and Shopping Agents}

Geoffrion and Krishnan (2001) have summarized the prospects for operations research in the electronic commerce era. MCDM/MAUT plays an important role in many of these developments. We point out two novel application areas for MCDM/MAUT: multiattribute online auctions, and comparison shopping agents. Both topics are widely discussed in the popular press and the academic literature.

There is an increasing awareness that price-only auctions are overly simplistic for most realworld purchasing situations. One cannot necessarily ignore the attributes of alternatives such as quality, terms of delivery, and warranty, which naturally leads to the definition of multi-attribute auctions. This 
notion has been explored in the context of a reverse auction by Chen-Ritzo et al. (2005) and Teich et al. (2004).

A central issue underlying multiattribute auctions is the elicitation of the auction owner's preferences over the relevant attributes/issues. Teich et al. (2004) outline approaches for accomplishing this task, including (a) eliciting a value function, (b) using the auction owner specified preference path, and (c) 'pricing out' (or 'costing out') all other dimensions (attributes) besides price and quantity. Approaches (a) and (c) have been implemented in web-based auction systems. The auction owner specified preference path is a simple, untested idea. Teich et al. (2004) recommend the use of 'pricing out' in the context of online auctions, since this dramatically simplifies the auctions, effectively reducing bids to quantity-price pairs in multi-unit auctions. In fact, 'pricing out' is an old technique often used by practicing decision analysts (see Keeney and Raiffa, 1976).

Early shopping agents were designed to find the 'best' deals for buyers, focusing solely on the price of the merchandise. Given that all the price information was posted on the Internet, the shopping agents would find the 'best' deals, and consumers would have no reason to buy elsewhere. As a result, many feared price wars. These price wars did not occur, however, because purchase decisions are based not only on price, but also on other attributes related to notions of quality and service. More sophisticated shopping agents, incorporating buyer's preferences over multiple attributes are being developed and evaluated (Sim and Choi, 2003). For example, the Tete-a-Tete shopping agent models and uses the buyer’s utility function (Maes et al., 1999).

Active Decisions (www.activebuyersguide.com) provides a sophisticated example of the implementation of MCDM/MAUT concepts in the business-to-consumer environment, and offers decision support for a large number of products and services. The web site allows consumers to select from a list of possible product attributes and create a multi-attribute preference model to rank order alternatives and facilitate comparisons. When utilizing the Decision Guide (as of September 20, 2003) to analyze digital cameras, the user could consider eleven possible product attributes. A decision maker 
could choose a subset of product attributes from this list, and then answer a series of questions to determine the weights on the selected attributes.

\section{Geographic Information System}

Many real-world spatial planning and management problems give rise to MCDM problems based on the use of Geographic Information Systems (GIS). Examples include environmental planning, urban planning, facilities location, real estate, and retailing. In fact, the Geographic Information and Multicriteria Decision Analysis (GIMDA) research group and the online Journal of Geographic Information and Decision Analysis (GIDA) have been established to explore the links and synergies between MCDM/MAUT and GIS. The GIS technology offers unique capabilities for analyzing spatial decision problems and handling spatial data. MCDM/MAUT, on the other hand, offers useful tools and concepts that incorporate preferences into GIS-based decision making. As the mission of the GIMDA research group states, there is a need for developing a unified framework for GIS-based MCDM/MAUT decision making. For further information, see the GIDA Journal or the book by Malczewski (1999).

\section{Engineering Application}

MCDM/MAUT is used in many fields of engineering. Often, however, the application of MCDM tools in engineering is based on simple scoring models. Examples of scholarly applications include river basin development and management, water regulation, chemical process optimization, aircraft wing design, forest management, environmental planning and management, and radiation therapy planning. See the Systems Analysis Laboratory web-page at the Helsinki University of Technology (http://www.sal.hut.fi/)

for several such MCDM/MAUT applications and Coello and Lamont (2004) for EMO applications. Also see Hobbs and Meier (2000) for an extensive coverage of the use of MCDM/MAUT methods in energy and environmental decisions.

Sophisticated applications of MAUT have appeared in the military/nuclear energy sector, notably related to the critical issues associated with nuclear weapons. Von Winterfeldt and Schweitzer (1998) evaluated alternatives for the replenishment of tritium in the U.S. nuclear weapons stock pile. Ten alternatives were evaluated based on the criteria of production assurance, cost, and environmental 
impacts. This analysis was influential in supporting the final recommendation by the U.S. Secretary of Energy.

In related work, a team of U.S. decision analysts was commissioned by the Department of Energy's Office of Fissile Materials Disposition to develop a multi-attribute utility model to evaluate alternatives for disposing excess plutonium. Subsequent to the U.S. study, Russian scientists modified the model with the aid of the U.S. team, and used it to evaluate Russian disposition alternatives (Butler et al., 2005). The MAUT analysis also highlighted the desirability of parallelism between U.S. and Russian plutonium disposition technologies. The Russians have decided to replicate the design of the US disposition facility in Russia, contributing to the synergy in the disposition policies.

Furthermore, many papers deal with relating a product's design parameters to performance measures. Typically, a value function is optimized. Köksalan and Plante (2003) have proposed a method that accounts for multiple criteria (maximization of process yield, maximization of process capability, minimization of process costs) via a procedure that interacts with and relies on the preferences of a decision maker. The procedure blends ideas from research in multiple-response design, multiple criteria optimization, and global optimization. According to the authors, the concepts and methods developed can be applied to problems such as supply chain management and multidisciplinary design optimization.

\section{Areas for Future Research}

The penetration of MCDM/MAUT concepts to the areas indicated above continues at an increasing rate, providing excellent opportunities for involvement for MCDM/MAUT researchers. The previous section commented on past research as well as ongoing and future research. In many cases the Internet will continue to provide additional challenges and opportunities. In this section, we identify several other areas with the potential for interesting future research.

\section{Mental Models}

One attractive area is to develop mental models of decision problems that relate a decision maker's perception of a problem to her fundamental objectives. In his work on "value-focused thinking”, Keeney (1992) made the distinction between a hierarchy of fundamental objectives linked to preferences, and 
models of interactions of attributes of an alternative that might be used to predict the performance of an alternative on each of these attributes. Keeney identified the latter as "means-ends" models to distinguish them from true preference models. The idea of "means-ends” models was anticipated by Keeney and Raiffa (1976) in their discussion of "proxy attributes." They noted that several attributes may relate to one fundamental objective, and suggested that the decision maker will have to think hard about these interactions when assessing attribute weights. However, they did not address the question of how these “mental models” of impacts of attributes on objectives might be quantified.

For situations with discrete levels of both attributes and objectives, Nadkarni and Shenoy (2001, 2004) provide an assessment procedure using a causal mapping approach to constructing Bayesian networks. The advantage of their procedure is that it explicitly measures uncertainty inherent in mental models, but the method becomes complex if the scale of measurement is not discrete. When the objectives and attributes are measured on a continuous scale, we need to ask a decision maker how much "impact" a criterion has on an objective. For example, the "appearance" of a car may have some "impact" on the objective of Prestige but not on the objective of Minimum Cost. This is a different notion from preference, but one that could be modeled, is intuitive, and could be measured on a ratio scale.

Ideas and tools that might be used for explicit assessment of means-ends models include conjoint analysis or statistical models to estimate tradeoffs or "weights” among attributes. Direct assessments by subjects might also be used, and it is worthwhile to note that such assessments may be ratio judgments, since the notion of an impact does have a natural zero and therefore a natural ratio scale. This perspective may provide a link between multiattribute utility theory and the AHP, if the AHP is viewed as measuring the relative "impacts" of attributes on objectives. Some efforts have been made to link these concepts to MCDM/MAUT models, as discussed by Montibeller et al. (2005) and by Butler et al. (2006).

\section{Revisiting Targets}

Another emerging area is the use of targets in problems involving multiple criteria. The idea is that the decision maker's utility or value may not depend on the levels of performance on different criteria, but instead on whether the levels meet a target or threshold on one or more criteria. MCDM scholars have 
been aware of this notion for many years; the notion provides a basis for goal programming. In recent years, several contributions to MAUT have examined this idea in more detail, particularly in situations where performance and goal levels themselves may be uncertain. For example, typical attributes in new product choice may be cost, quality, and different features, and the corresponding targets may be the best performance on these attributes relative to the competition: see Bordley and Kirkwood (2004) and Tsetlin and Winkler (2006). It would seem natural to revisit MCDM models involving goals or targets for attributes in light of these new studies. Related issues that could be addressed would be methods for selecting targets in ways that are incentive compatible in organizations.

\section{Decision Support in a Distributed Environment}

Decision support in a distributed environment is somewhat different from what we had expected in the early 1990s. For example, what is a user? Our fields have traditionally sought to support corporate managers. However, household consumers need support for purchasing decisions. What kind of decision support do they want in an Internet or mobile environment? The problem may not be one of having insufficient information, but rather one of having ‘too much' or an unknown quality of information. We may have to filter information. This is a potential application and development area for MCDM/MAUT. Quadratic and Stochastic Programming

Significant progress may be anticipated in computing the nondominated set of a multiple objective problem that is linear, with the exception of one or two quadratic objectives. Instead of the nondominated set consisting of flat faces as in an MOLP, from the research of Steuer et al. (2005) we know that the nondominated set is made up of paraboloidic platelets. With an algorithm possible for computing all such platelets, combined with the option to populate each platelet with additional points, a densely populated discretized representation of the nondominated set of such a problem will soon be possible. The task of finding the most preferred nondominated point will then be similar to finding the best in a discretized nondominated set produced by an evolutionary algorithm.

The capability to compute nondominated sets for "almost-linear" problems should allow MCDM/MAUT to move into the area of multi-objective stochastic programming, where the quadratic 
objectives might represent variance (Caballero et al., 2001). This should open up multi-objective stochastic programs to new multiple criteria applications in finance, as suggested for instance by Aouni et al. (2006), Bana e Costa and Soares (2001), and Hallerbach and Spronk (2002).

\section{Usefulness of Dotted Representations of Nondominated Sets}

Since the early days of MCDM, there have basically been two approaches for solving multiple criteria optimization problems. One is to compute a representation of the nondominated set, often in the form of “dots" (i.e., discrete solutions), and then spend time searching the representation for a final solution. The other is to accumulate knowledge about the nondominated set by sequentially probing or sampling from the set until a final solution can be identified. With improved computers and algorithms we see the two approaches blending together. When dotted representations involve many points, interactive procedures can be modified to sample from large sets of given points as effectively as from nondominated sets only known implicitly, and for which they were originally designed. Packages such as MATLAB take only a few seconds to identify which vector out of a million is closest to another vector. Benson and Sun (2000) and Sayin (2003) have worked on new methods that might be able to obtain dotted representations of the nondominated sets of large MOLPs in reasonable time.

\section{Evolutionary Multi-Objective Optimization}

We anticipate that EMO and MCDM/MAUT will move closer together to enhance both research programs. The main thrust of EMO research and application has been to find well-distributed sets of nondominated solutions for problems involving only a few (often just two) objectives. When efforts are made to develop well-distributed sets of nondominated solutions for problems involving more objectives, EMO methodologies and MCDM/MAUT techniques may be blended in a synergistic manner.

There are at least two ways to do this. First, an EMO may be applied to generate a set of nondominated solutions, and then an MCDM/MAUT procedure may be used to choose a most preferred solution from the set. This process is, however, rather labor intensive. Another way is to embed MCDM/MAUT principles within an EMO through a utility or fitness measure, so that the interaction with 
the decision maker commences right from the beginning of the solution process. See Pamuk and Köksalan (2003), Tan et al. (2005), Köksalan and Pamuk (2007), and Deb and Sundar (2006).

Collaborative efforts between EMO and MCDM/MAUT researchers, notably the Dagstuhl Conferences, have begun to focus on using ideas from both fields. When solving higher-dimensional problems, more effective visualization techniques must also be developed to represent multi-objective data to the decision maker. For this problem, the EMO and MCDM/MAUT communities may collaborate in a mutually beneficial manner. Lotov et al. (2004) provide possibly useful ideas.

\section{Challenges from Practice}

Kasanen et al. (2000) studied several real-world managerial decision situations, and drew conclusions for MCDM/MAUT research. Without making claims of being “representative”, the authors felt that the processes they studied were not uncommon in the real world. In the absence

of a formalized decision process, decision makers did not know or generate all, or even a reasonable subset of options, evaluate consequences in terms of explicitly stated criteria, and make truly informed decisions. Even if aided by formal decision models, decision makers may not want to or may not be able to act according to the MCDM/MAUT paradigm. Hence there may be good reasons to broaden the MCDM/MAUT framework to better reflect real-world decision processes, as described by organizational theorists.

\section{Conclusions}

We believe that the conclusions of Dyer et al. (1992) are still valid. Extensive research has been done in the fields of MCDM, MAUT, and related decision support systems in the past decade, as documented by our bibliometric analysis. Yet additional work remains to be done. The Internet will continue to provide challenges for the MCDM/MAUT research community. It will also continue to be a distribution outlet for research and software. We also envision that several subfields which developed rather independently, such as EMO, Goal Programming, and multi-objective Decision Analysis will provide opportunities for 
collaboration with MCDM/MAUT researchers, leading to synergistic advances and less fragmentation of these fields.

\section{References}

Aouni, B., F. Ben Abdelaziz and R. El-Fayedh. 2006. Chance Constrained Compromise Programming for Portfolio Selection. Laboratoire LARODEC, Institut Superieur de Gestion, Tunis, Tunisia.

Bana e Costa, C. A. and J. O. Soares (2001), Multicriteria Approaches for Portfolio Selection: An Overview, Review of Financial Markets 4 19-26.

Belton, V. 1992. Integrating Data Envelopment Analysis with Multiple Criteria Decision Analysis. In Goicoechea, A., L. Duckstein and S. Zionts (Eds.), MCDM, Springer, Berlin, 71-79.

Benson, H. P. and E. Sun. 2000. Outcome Space Partition of the Weight Set in Multiobjective Linear Programming. Journal of Optimization Theory and Applications 105 17-36.

Bordley, R. and C. W. Kirkwood. 2004. Multiattribute Perference Analysis with Performance Targets. Operations Research 52 823-835.

Branke, J., T. Kaussler and H. Schmeck. 2001. Guidance in Evolutionary Multi-Objective Optimization. Advances in Engineering Software 32 499-507.

Butler, J., A. Chebeskov, J. S. Dyer, T. Edmunds, J. Jia and V. Oussanov. 2005. The Use of MultiAttribute Utility Theory for the Evaluation of Plutonium Disposition Options in the United States and Russia. INTERFACES 35 88-101.

Butler, J., J. Dyer, and J. Jia. 2006. Using Attributes to Predict Objectives in Preference Models. Decision Analysis 3 100-116.

Caballero, R., E. Cerdá, M. M. Muñoz, L. Rey and I. M. Stancu-Minasian (2001), “Efficient Solution Concepts and their Relations in Stochastic Multiobjective Programming,” Journal of Optimization Theory and Applications 110 53-74.

Caballero, R., T. Gómez and F. Ruiz. 2007. Goal Programming: Realistic Targets for the Near Future. Unpublished Manuscript, Department of Economics, University of Malaga, Malaga, Spain. 
Carlson, K. and L. Pearo. 2004. Limiting Predecisional Distortion by Prior Valuation of Attribute Components. Organizational Behavior and Human Decision Processes 94 48-59.

Carlson, K. and S. Bond. 2006. Improving Preference Assessment: Limiting the Effect of Context through Pre-exposure to Attribute Levels. Management Science 52 410-421.

Charnes, A. and W. W. Cooper. 1961. Management Models and Industrial Applications of Linear Programming. Wiley, New York.

Charnes, A., W. W. Cooper and E. Rhodes. 1978. Measuring Efficiency of Decision Making Units. EJOR $2429-444$.

Chen-Ritzo, C., T. Harrison, A. Kwasnica and D. Thomas. 2005. Better, Faster, Cheaper: An Experimental Analysis of a Multiattribute Reverse Auction Mechanism with Restricted Information Feedback. Management Science 51 1753-1762.

Coello, C.A.C. and G.B. Lamont. 2004. Applications of Multi-Objective Evolutionary Algorithms. World Scientific.

Corne, D., J. Knowles and M. Oates. 2000. The Pareto Envelope-Based Selection Algorithm for Multiobjective Optimization. Proceedings of the Sixth International Conference on Parallel Problem Solving from Nature VI (PPSN-VI), 839-848.

Deb, K. 2001. Multi-Objective Optimization Using Evolutionary Algorithms. Wiley, New York.

Deb, K. 2003. Unveiling Innovative Design Principles by Means of Multiple Conflicting Objectives. Engineering Optimization 35 445-470.

Deb, K. and H. Gupta. 2005. Searching for Robust Pareto-Optimal Solutions in Multi-Objective Optimization. Proceedings of the Third Evolutionary Multi-Criterion Optimization (EMO-03).

Deb, K. and Sundar, J. 2006. Reference point based multi-objective optimization using evolutionary algorithms. Proceedings of the Genetic and Evolutionary Computation Conference (GECCO2006). New York: The Association of Computing Machinery (ACM), 635-642Conference, Lecture Notes in Computer Science, 3410, Springer-Verlag, 150-164. 
Delquié, P. 1993. Inconsistent Trade-offs between Attributes: New Evidence in Preference Assessment Biases. Management Science 39 1382-1395.

Delquié, P. 1997. Bi-matching: A New Preference Assessment Method to Reduce Compatibility Effects. Management Science 43 640-658.

Doumpos, M. and C. Zopounidis. 2002. Multicriteria Decision Aid Classification Methods. Kluwer Academic Publishers.

Doyle, R. H. and J. R. Green. 1993, DEA and MCDM, Omega 6 713-715.

Dyer, J. S., P. C. Fishburn, R. E. Steuer, J. Wallenius and S. Zionts. 1992. Multiple Criteria Decision Making, Multiattribute Utility Theory: The Next Ten Years. Management Science 38 645-654.

Edwards, W., R. Miles and D. von Winterfeldt (Eds.). 2007. Advances in Decision Analysis. Cambridge University Press, forthcoming.

Ehrgott, M. 2005. Multicriteria Optimization, Springer-Verlag.

Ehrgott, M. and X. Gandibleux. 2000. An Annotated Bibliography of Multiobjective Combinatorial Optimization. OR Spektrum 22 425-460.

Ehtamo, H. and R. P. Hämäläinen. 2001. Interactive Multiple-Criteria Methods for Reaching Pareto Optimal Agreements in Negotiations. Group Decision and Negotiation 10 475-491.

Ehtamo, H., R. P. Hämäläinen, P. Heiskanen, J. Teich, M. Verkama and S. Zionts. 1999, Generating Pareto Solutions in Two-Party Negotiations by Adjusting Artificial Constraints, Management Science 45 1697-1709.

Figueira, J., S. Greco and M. Ehrgott (Eds.). 2005. Multiple Criteria Decision Analysis: State of the Art Surveys. Kluwer Academic Publishers.

Fisher, R. 1978. International Mediation: A Working Guide. International Peace Academy, New York.

Fonseca, C. M. and P. J. Fleming. 1993. Genetic Algorithms for Multiobjective Optimization: Formulation, Discussion, and Generalization. Proceedings of the Fifth International Conference on Genetic Algorithms, 416-23. 
Fonseca, C., P. J. Fleming, E. Zitzler, K. Deb and L. Thiele (Eds.). 2003. Proceedings of the Second EMO-03 Conference. Lecture Notes in Computer Science, 2632, Springer.

FORTUNE Magazine, “Staying Smart”, May 10, 1999.

Gardiner, L. R. and R. E. Steuer. 1994. Unified Interactive Multiple Objective Programming. EJOR 74 391-406.

Geoffrion, A. and R. Krishnan. 2001. Prospects for Operations Research in the E-Business Era. INTERFACES 30 6-36.

Greco, S., B. Matarazzo and R. Slowinski. 1999. The Use of Rough Sets and Fuzzy Sets in MCDM. In T. Gal, T. Stewart, and T. Hanne (eds.), Advances in Multiple Criteria Decision Making, Kluwer Academic Publishers, Dordrecht, 14.1-14.59.

Greco, S., B. Matarazzo and R. Slowinski. 2005. Decision Rule Approach. In J. Figueira, S. Greco and M. Ehrgott (eds.), Multiple Criteria Decision Analysis: State of the Art Surveys, Springer, New York, 507-608.

Hallerbach, W. G. and J. Spronk. 2002. The Relevance of MCDM for Financial Decisions. Journal of Multi-Criteria Decision Analysis 11 187-195.

Halme, M., T. Joro, P. Korhonen, S. Salo and J. Wallenius. 1999. A Value Efficiency Approach to Incorporating Preference Information in Data Envelopment Analysis. Management Science 45 103-115.

Hammond, J. S., Keeney, R.L., and Raiffa, H. 1998. Even swaps: A rational method for making trade-offs. Harvard Business Review 76 137-149.

Hobbs, B. F. and P. Meier. 2000. Energy Decisions and the Environment: A Guide to the Use of Multicriteria Methods. Kluwer Academic Publishers.

Hogarth, R. and N. Karelaia. 2005. Simple Models for Multiattribute Choice with Many Alternatives: When It Does and Does Not Pay to Face Trade-offs with Binary Attributes. Management Science 51 1860-1872. 
Horn, J., N. Nafploitis and D. Goldberg. 1995. A Niched Pareto Genetic Algorithm for Multi-Objective Optimization. Proceedings of the First IEEE Conference on Evolutionary Computation 82-87.

Hämäläinen, R.P. 2004. Reversing the Perspective on Applications of Decision Analysis. Decision Analysis 1 26-31.

Hämäläinen, R.P. and S. Alaja. 2003. The Threat of Biases in Environmental Decision Analysis. Research Reports, E12, Systems Analysis Laboratory, Helsinki, Finland, www.e-reports.sal.hut.fi.

Ignizio, J. and C. Romero. 2003. Goal Programming. In H. Bidgoli (Ed.), Encyclopedia of Information Systems. Academic Press 2 489-500.

Jaszkiewicz, A. 2001. Multiple Objective Metaheuristic Algorithms for Combinatorial Optimization. Habilitation Thesis, 360, Poznan University of Technology, Poznan.

Joro, T., P. Korhonen and J. Wallenius. 1998. Structural Comparison of Data Envelopment Analysis and Multiple Objective Linear Programming. Management Science 44 962-970.

Kasanen, E., H. Wallenius, J. Wallenius and S. Zionts. 2000. A Study of High-Level Managerial Decision Processes, with Implications for MCDM Research. EJOR 120 496-510.

Keefer, D., C. W. Kirkwood and J. Corner. 2004. Perspective on Decision Analysis Applications. Decision Analysis 1 4-22.

Keeney, R. 1992. Value-Focused Thinking: A Path to Creative Decision Making. Harvard University Press, Cambridge, Massachusetts.

Keeney, R. 2002. Common Mistakes in Making Value Trade-offs. Operations Research 50 935-945.

Keeney, R. and H. Raiffa. 1976. Decisions with Multiple Objectives: Preferences and Value Tradeoffs. Wiley, New York.

Kirkwood, C. W. 1997. Strategic Decision Making: Multiobjective Decision Analysis with Spreadsheets. Duxbury Press, Belmont, California.

Korhonen, P. and J. Karaivanova. 1999. An Algorithm for Projecting a Reference Direction onto the Nondominated Set of Given Points. IEEE Trans. on Systems, Man, and Cybern. A 29 429-435. 
Korhonen, P. and M. Syrjänen. 2004. Resource Allocation Based on Efficiency Analysis. Management Science 50 1134-1144.

Korhonen, P. and J. Wallenius. 1996. Letter to the Editor: Behavioral Issues in MCDM: Neglected Research Questions. Journal of Multi-Criteria Decision Analysis 5 178-182.

Kouvelis, P. and S. Sayin. 2006. Algorithm Robust for the Bicriteria Discrete Optimization Problem: Heuristic Variations and Computational Evidence. Annals of Operations Research 147 71-85.

Köksalan, M. and S. Pamuk. 2007. An Evolutionary Metaheuristic for Approximating PreferenceNondominated Solutions. INFORMS Journal on Computing, forthcoming.

Köksalan, M. and R. D. Plante. 2003. Interactive Multi-Criteria Optimization for Multiple Response Product and Process Design. Manufacturing and Service Operations Management 5 334-347.

Liesiö, J., P, Mild and A. Salo. 2007. Preference Programming for Robust Portfolio Modeling and Project Selection. EJOR 181 1488-1505.

Lotfi, V., T. Stewart and S. Zionts. 1992. An Aspiration-Level Interactive Model for Multiple Criteria Decision Making. Computers \& Operations Research 19 671-681.

Lotov, A., V. A. Bushenkov, and G. K. Kamenev. 2004. Interactive Decision Maps: Approximation and Visualization of Pareto Frontier. Kluwer Academic Publishers.

Maes, P., R. Guttman, and A. Moukas. 1999. Agents that Buy and Sell. Communications of the ACM 42 (3) 81-ff.

Malczewski, J. 1999. GIS and Multicriteria Decision Analysis. Wiley, New York.

Matheson, J. and D. Matheson. 2007. Organizational Decision Analysis. In W. Edwards, R. Miles and D. von Winterfeldt (Eds.), Advances in Decision Analysis, Cambridge Univ. Press, forthcoming.

Miettinen, K. 1999. Nonlinear Multiobjective Optimization. Kluwer Academic Publishers.

Miettinen, K. and M. M. Mäkelä. 1995. Interactive Bundle-Based Method for Nondifferentiable Multiobjective Optimization: NIMBUS. Optimization 34 231-246. 
Montibeller, G., V. Belton, F. Ackermann and L. Ensslin. 2005. Reasoning Maps for Decision Aid: An Integrated Approach for Problem-Structuring and Multi-Criteria Evaluation. Management Science WP 2005/11, Kingston Business School, London.

Nadkarni, S. and P. Shenoy. 2001. A Bayesian Network Approach to Making Inferences in Causal Maps. EJOR 128 479-498.

Nadkarni, S. and P. Shenoy. 2004. A Causal Mapping Approach to Constructing Bayesian Networks. Decision Support Systems 38 259-281.

Olson, D. 1996. Decision Aids for Selection Problems. Springer-Verlag.

Pamuk, S. and M. Köksalan. 2003. An Interactive Evolutionary Metaheuristic for Multiobjective Combinatorial Optimization. Management Science 49 1726-1738.

Pawlak, Z. 1982. Rough Sets. International Journal of Computer \& Information Science 11 341-356.

Pöyhönen, M. and R. P. Hämäläinen. 2001. On the Convergence of Multiattribute Weighting Methods. EJOR 129 569-585.

Ragsdale, C. T. 2004. Spreadsheet Modeling and Decision Analysis: A Practical Introduction to Management Science. South Western College Publishing.

Raiffa, H. 1982. The Art and Science of Negotiation. Harvard University Press.

Roy, B. 1998. A Missing Link in Operational Research Decision Aiding: Robustness Analysis. Foundations of Computing and Decision Sciences 23 141-160.

Saaty, T. L. 1999. Decision Making for Leaders, RWS Publications, Pittsburgh.

Salo, A. and R.P. Hämäläinen. 1992. Preference Assessment by Imprecise Ratio Statements. Operations Research 40 1053-1061.

Sayin, S. 2003. A Procedure to Find Discrete Representations of the Efficient Set with Specified Convergence Errors. Operations Research 51 427-436.

Schaffer, J. D. 1984. Some Experiments in Machine Learning Using Vector Evaluated Genetic Algorithms. PhD Thesis, Vanderbilt University, Nashville, Tennessee. 
Scheubrein, R. and S. Zionts. 2006. A Problem Structuring Front End for a Multiple Criteria Decision Support System. Computers \& Operations Research 33 18-31.

Sebenius, J. K. 2007. Negotiation Analysis: Between Decisions and Games. In W. Edwards, R. Miles and D. von Winterfeldt (Eds.), Advances in Decision Analysis, Cambridge Univ. Press, forthcoming.

Sim, K. M. and C. Y. Choi. 2003. Agents that React to Changing Market Conditions. IEEE Transactions on Systems, Man, and Cybernetics B 33 188-201.

Slowinski, R. 1992. Intelligent Decision Support: Handbook of Applications and Advances of the Rough Sets Theory. Kluwer Publishers.

Slowinski, R., S. Greco, and B. Matarazzo. 2002. Axiomatization of Utility, Outranking and DecisionRule Preference Models for Multiple-Criteria Classification Problems under Partial Inconsistency with the Dominance Principle. Control and Cybernetics 31 1005-1035.

Srinivas, N. and K. Deb. 1994. Multi-Objective Function Optimization Using Non-dominated Sorting Genetic Algorithms. Evolutionary Computation Journal 2 221-248.

Statnikov, R. B. and J. B. Matusov. 2002. Multicriteria Analysis in Engineering. Kluwer Publishers.

Steuer, R. E. 2006. ADBASE: A Multiple Objective Linear Programming Solver for All Efficient Extreme Points and Unbounded Efficient Edges. Terry College of Business, University of Georgia, Athens, Georgia.

Steuer, R. E., Y. Qi, and M. Hirschberger. 2005. Multiple Objectives in Portfolio Selection,” Journal of Financial Decision Making 1 5-20.

Sun, M. 2003. Procedures for Finding Nondominated Solutions for Multiple Objective Network Programming Problems. Transportation Science 37 139-152.

Tan, K. C., E. F. Khor and T. H. Lee. 2005. Multiobjective Evolutionary Algorithms and Applications, Springer, Berlin.

Teich, J., H. Wallenius and J. Wallenius. 1994. Advances in Negotiation Science. Transactions on Operational Research 6 55-94. 
Teich, J., H. Wallenius, J. Wallenius and O. Koppius. 2004. Emerging Multiple Issue e-Auctions. EJOR 159 1-16.

Tsetlin, I. and R. Winkler. 2006. Decision Making with Multiattribute Performance Targets: The Impacts of Changes in Performance and Target Distributions. Working Paper, Duke University.

Ulungu, E. and J. Teghem. 1994. Multiobjective Combinatorial Optimization Problems: A Survey. Journal of Multi-Criteria Decision Analysis 3 83-104.

Von Winterfeldt, D. and E. Schweitzer. 1998. An Assessment of Tritium Supply Alternatives in Support of the U.S. Nuclear Weapons Stockpile. INTERFACES 22 92-118.

Wang, J. G. and S. Zionts. 2005. WebAIM: An Online Aspiration Level Interactive Method. Journal of Multi-Criteria Decision Analysis 13 51-63.

Wang, J. G. and S. Zionts. 2006. The Aspiration Level Interactive Method (AIM) Reconsidered: Robustness of Solutions to Multiple Criteria Problems. EJOR 17 (2) 948-958.

Wang, J. G. and S. Zionts. 2007. Negotiating Wisely: Considerations Based on MCDM/MAUT. Forthcoming in EJOR.

Wiecek, M. M. and H. Zhang. 1997. A Parallel Algorithm for Multiple Objective Linear Programs. Computational Optimization and Applications 8 41-56.

Yang, J. B. and D. Li. 2002. Normal Vector Identification and Interactive Tradeoff Analysis Using Minimax Formulation in Multiobjective Optimization. IEEE Transactions on Systems, Man, and Cybernetics A 32 305-319.

Zitzler, E., K. Deb, L. Thiele, C. A. C. Coello and D. Corne. 2001. Proceedings of the First Evolutionary Multi-Criterion Optimization Conference, Lecture Notes in Computer Science, 1993, Springer, New York. 